\title{
Nursing Care for Adolescent Idiopathic Spine Patients
}

\section{Phyllis D'Ambra}

Children's Orthopaedic Center, Children's Hospital Los Angeles, Los Angeles, CA 90027, USA

Abstract

Background: Nursing care for spine patients with adolescent idiopathic scoliosis requires education, plan of care, and involvement of a multi-disciplinary team.

\section{Publication History:}

Received: May 08, 2018

Accepted: August 04, 2018

Methods: To be a valuable member of the team, nurses caring for the spine patient must learn about the Published: August 06, 2018 care, operative procedure and outcomes.

Results: Nursing intervention can help facilitate patients and families to navigate the hospital course and Keywords: can reduce the length of a patient's hospital stay.

Conclusion: Well informed members of a nursing team can assist families and patients, resulting in reduced anxiety and expedite a patient's return to daily activities.

Adolescent Idiopathic Scoliosis (AIS) is more than merely a lateral and rotational deviation of the spine. From diagnostic testing to surgical correction, the treatment of AIS is a multidisciplinary approach which demands the skills of all involved to achieve the desired outcome. Review of the pathophysiological course of scoliosis reveals the complexity of its development, causation, leading to a better understanding of the history of scoliosis. Structural scoliosis is not confined to adolescents but in this case, was our study population. Through a careful review and evaluation, nursing can help the family and patient navigate the complex system of hospitalization, surgical intervention, recovery and transition back home.

The treatment of scoliosis can span a number of years starting with non-operative treatment to a spinal fusion surgery. Living with this condition requires sacrifice and cooperation of all members of the family unit and others with whom the patient interacts. A healthy adolescent is a melting pot of sociological, economic, psychological and cultural conflicts encased in an unfamiliar body. Coupled with the changes in body image due to scoliosis, they can become impetuous and vacillating in response to unaccepting adults and at times, an unacceptable body image. Insatiable energy and an appetite for life, experimentation and drive often collide with changes in body image. The indestructible adolescent travels a faltering path towards achievement of adult maturity. Superimposed upon the normal turmoil of growing up with scoliosis can be perceived as an insurmountable burden. Only if the team understands and accepts the implications of the impact of scoliosis on the adolescent can they provide effective guidance to the patient and family.

The design of the Nursing Care Standard is a means to identify nursing problems and establish a plan of care. The anticipated outcome criteria that required surgical intervention: identify preoperative potential psychological, social and physical barriers to surgery. The adolescent has concerns about body image and a fear of the unknown. The goal of care for a patient with adolescent idiopathic scoliosis is to create an environment that allows expression of fear, verbalization of their concerns and understanding of the surgical intervention. Family members and core support groups will also need to be well informed and to express their fears and concerns. The nurse's role in the navigation of the patient through surgery for scoliosis is pivotal in the success of their surgical plan.

The surgeon provides a detailed explanation of surgery, potential risks and plan of recovery. The nurse then prepares the patient and family for preoperative testing and preparation, hospital course and home care. There are several modalities that we have found at
Children's Hospital Los Angeles (CHLA) to insure that patients and families are fully aware of their commitment to the treatment plan. These include preoperative education and planning materials that reduce the risk of misunderstanding and complications. The patient and families are provided with all the necessary information at their preoperative visit by the nurse who is then reinforced by the Spine Fusion and Recovery Brochure. We also provide a specialized spine class in the evening for patients and families. The class is a comprehensive review of the preoperative preparation, hospital course including all equipment used and home care. We have an in depth discussion about the anatomy and physiology of scoliosis. We also discuss the emotional recovery. Additionally, patients and their families are told the goal is to be discharged in three days and given detailed handouts which include information on pain management. This forum also gives the patient, members of the family and friends the opportunity to ask questions and express their concerns. We recognize one of the outcomes that benefits patients is their awareness of other patients and families that are having the same experience.

The implementation of a Nursing Care Standard for our patients through protocol/expectations has helped reduce our patients' hospital stay from 5 to 7 days to 3 to 3.5 days [1]. The accelerated discharge protocol used by the team embraces a variety of modalities such as nursing, physical therapy, pain management and orthopaedic surgery to help facilitate this outcome [1]. The nursing team in the inpatient unit has been the driver designing a protocol to facilitate 3 to 3.5 day discharge [2] (Figure 1).

Presented with the complexity of surgical intervention in the treatment of scoliosis, the nurse becomes acutely aware of the importance of first line observation. Management of the patient in any phase of the treatment demands knowledge and skills in order for complications to be avoided and therapeutic effects be achieved. From the first encounter with the patient, the nursing approach determines the degree of participation of patient and their understanding and cooperation.

"Corresponding Author: Phyllis D'Ambra, RN, MPA, Children's Orthopaedic Center, Children's Hospital Los Angeles, Los Angeles, CA 90027, USA; E-mail: pdambra@chla.usc.edu

Citation: D'Ambra P (2018) olescent Idiopathic Spine Patients. Int J Pediatr Neonat Care 4: 141. doi: https://doi.org/10.15344/2455-2364/2018/141

Copyright: ( 2018 D’Ambra. This is an open-access article distributed under the terms of the Creative Commons Attribution License, which permits unrestricted use, distribution, and reproduction in any medium, provided the original author and source are credited. 


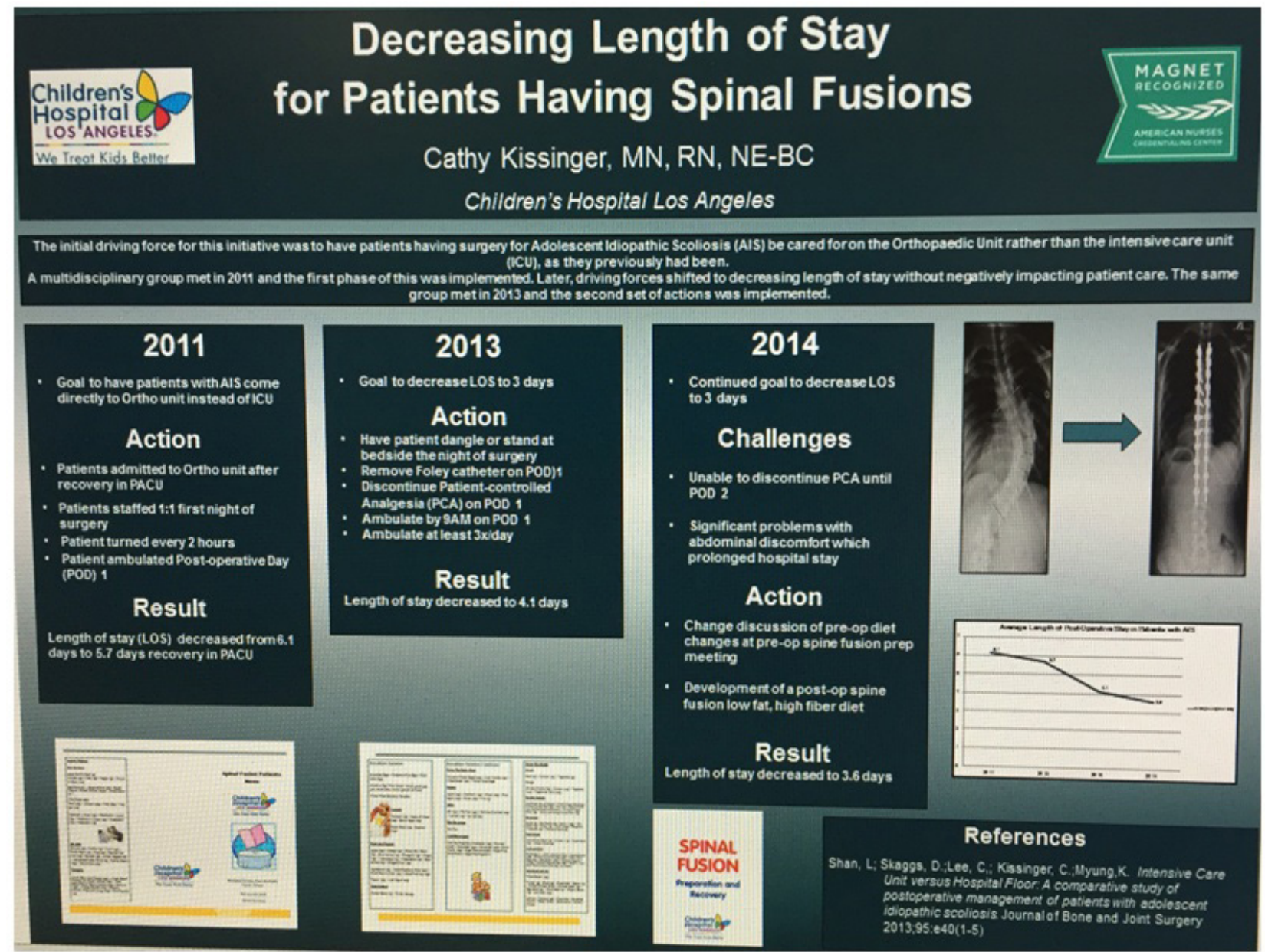

Figure 1: Decreasing length of stay for patients having spinal fusions.

Reduction of fear through well thought out orientation can ease what can otherwise become a very traumatic experience. Physiological responses have a limited relationship to compliance: stressful experiences do not elicit patient compliance and wellness. Surgical plan of care, which introduces sophisticated devices require nurses skill in the intricacies of observation, pain management problem solving. Corrective action must be superseded by correct application of nursing principles. Problems should be solved by appropriate and timely intervention. Nursing must encompass a body of knowledge that will supplement assessment and develop a standard of care for every individual patient and family constantly seeking to reassess the patient to anticipated outcomes.

Scoliosis, although a structural deformity, represents only a partial aspect of the total person for whom you have elected to provide care. Plan of action should be integrated, assessing that the surgical correction and the hospitalization are both seen by the patient and family as a growth experience.

\section{Competing Interests}

The authors declare that no competing interests is present.

\section{References}

1. Sanders AE, Andras LM, Sousa T, Kissinger C, Cucchiaro G, et al. (2017) Accelerated Discharge Protocol for Posterior Spinal Fusion Patients With Adolescent Idiopathic Scoliosis Decreases Hospital Postoperative Charges 22. Spine 42: 92-97.

2. Kissinger C (2017) Decreasing Length of Stay for Patients Having Spinal Fusions. ANCC National Magnet Conference. Houston, TX. 